\title{
Kommunikations-Prospektive und Aufgabe der Kirche in Lateinamerika
}

\author{
von Victorino Zecchetto
}

Das umfassende Thema Kommunikations-Prospektive bis zum Jahr 2000 und die Aufgabe der Kirche in Lateinamerika müssen wir notwendig auf einige grundlegende Aspekte begrenzen. Das erfordert die Darstellung auf wenigen Seiten. Darum beschränken wir uns auf eine knappe Zusammenstellung der Fakten um die Begriffe „Prospektive“, „Kommunikation" und "Lateinamerika“. Und wir zielen dabei eine zusammenfassende Überschau der Folgerungen für die Zukunft der Kirche des Subkontinents an.

\section{Die Prospektive}

Bis zum Jahre 1975 gab es in der Welt über 400 Zentren, die sich mit der Zukunft befaßten. Die Menschheit erlebt zur Zeit den Übergang in eine neue Dimension: die Zukunft. Die Aufgabe, die Zukunft anzugehen, kann auf zweierlei Weise erfolgen: entweder mit Hilfe der "Proferenz" oder mit Hilfe der „Prospektive“. Die „Proferenz" bedient sich der Techniken der Prognose, indem sie von der Vergangenheit und der Gegenwart ausgeht, um die Zukunft der Welt von morgen vorauszubestimmen. Die "Prospektive“ hingegen geht den umgekehrten Weg von der Zukunft zur Gegenwart. Man versetzt sich in eine wünschenswerte Zukunft mit dem Ziel, sich in der Gegenwart besser einzurichten und in der heutigen Wirklichkeit wirksamer vorzugehen. Die Prospektive ist nicht reine Utopie, sondern artikuliert konkrete Kräfte, um die Welt im Blick auf den "Pol“ der Zukunft umzugestalten. Nehmen wir etwa eine Prospektive vor bei einem Kommunikationsmittel, wie es die Zeitung darstellt. Statt auf den großen Rotationsmaschinen der Gegenwart wird die Tageszeitung künftig im eigenen Haus "gedruckt“. Das geschieht über Hertzwellen auf weißem Papier, in dessen Kern magnetischer Keramikstoff aufgelöst ist, und zwar so, daß nach Benetzen mit Wasser die von einer Zentralantenne übertragenen Druckzeilen sichtbar werden. Der Inhalt der Zeitung ändert sich fortlaufend mit der Folge der Nachrichten.

Diese „künftige“ Realität bedingt einen ungeheuren Wandel der Wirtschaftstrukturen: er trifft die Druckereien, Herstellungsstätten, Verteilungssysteme....

In dem Maß, wie sich der Wandel vollzieht, tauchen neue Organisationsformen der Gesellschaft und der Institutionen auf. Die tieferen Umgestaltungen vollziehen sich jedoch in den "Haltungen" der Menschen. In ihrem Innern werden die großen Schlachten der Prospektive entfesselt. Tatsächlich bedingt jeder beliebige Wandel, den die Zukunft aufzwingt, eine Entscheidung für das, was heute noch nicht vorhanden ist. Ein durch die Prospektive angezeigtes Künftiges muß, ehe es in Gang gesetzt wird, einen gewissen Grad von Annehmbarkeit genießen oder sich eines

Prof. Victorino Zecchetto SDB ist der Direktor des "Centro Salesiano de Audiovisuales“ in Quito, Ecuador, und Autor des Buches „Educación Catequesis Audiovisuales“. 
Wertausgleichs seitens der Menschen erfreuen, damit die Vorteile des Neuen und des Alten bedacht werden und so der Wille zum Wandel hinneigt.

Während auf technischem Gebiet der Wandel zum Fortschritt bis zu einem gewissen Punkt von der Werbung aufgedrängt wird, welche die Vorteile der in Vorschlag gebrachten neuen Modelle aufzeigt, liegt der Fall im menschlichen Bereich anders. Wenn wir "menschlichere" Existenzarten gestalten wollen, die den neuen Horizonten tiefgreifender Erwartungen entsprechen, dann muß ein qualitativer Sprung getan werden. Denken wir etwa nur an den Erziehungsbereich. In Lateinamerika prangerten bereits verschiedene Theoretiker der pädagogischen Wissenschaften die veraltete Schulstruktur in Sicht auf die prospektive Bestimmung der Erziehung an. „Wir dürfen nicht vergessen, daß die siebenjährigen Kinder, die heute die Grundschule besuchen, ihr Hochschulstudium am Vorabend des Jahres 2000 abschließen. Heute sind wir uns aber durchaus alle einig, daß das Leben im Jahre 2000 zutiefst von den heutigen Gewohnheiten abweichende Merkmale aufweisen wird." ${ }^{2}$ Mit anderen Worten, es bewahrheitet sich, was McLuhan bereits andeutete: „Die Schulinstitute verschwenden jeden Tag mehr und mehr Energie, um die Schüler auf eine Welt vorzubereiten, die schon nicht mehr existiert." Und trotzdem gibt es schulische Zukunftspläne mit Wahrscheinlichkeitsmerkmalen im Überfluß. Woran fehlt es da? An der Annahmebereitschaft für neue Modelle und am Handeln nach Vorschlag, obwohl unsere Gegenwart in Frage gestellt wird. Vor kurzem erfuhr ich, daß ein lateinamerikanisches Land für 26 Millionen Dollar Schulmaterial von hoher technischer Qualität zu kaufen plant. Wahrhaftig umstürzend! Aber es besteht nicht nur das Problem, daß es an Lehrern mangelt, die in der Lage sind, das Material gut einzusetzen, sondern vor allem ist die Annahmebereitschaft blockiert. Sehr wenige besitzen die Vorstellungskraft und das geistige Gespür für die Zukunft. Man mißt die Erziehung weiterhin an der Vergangenheit und prüft sie nicht vom erhöhten Standort der Zukunft aus.

Es besteht die Gefahr, daß Zwangsmaßnahmen der Behörden von oben erfolgen (vielfach aus volksfremden Erwägungen), man plant willkürlich und schafft so noch mehr Konflikte.

Wenn wir uns ein wenig weiter in Richtung Prospektive vorwagen, sehen wir, daß sie tiefere Probleme aufwirft. Heute hat man gewisse Modelle gewählt, deren künftiger Wahrscheinlichkeitsgrad sie als ungeheuer tunlich hinstellt. Die heutige Atomprogrammierung erfolgt nach leicht vorausschaubaren Phasen. Praktisch kann man Gegenwart und Zukunft zugleich abschätzen. So kann zum Beispiel die künftige Neutronenbombe Menschen töten, ohne Gebäude zu zerstören. Durch intensive Strahlung tötet die neue Bombe die Menschen und verursacht keine nennenswerte Wirkung an Gebäuden und Material ringsum. Wir können heute durchaus unterstellen, daß man angesichts der atomaren Prospektive, die uns die Vernichtung der Menschheit durch sie selber anzeigt, die Atomenergie nur für friedliche $Z$ wecke unter Kontrolle zu halten bereit ist; das würde aber einschließen, daß man die Verantwortlichen nicht nur zutiefst überzeugt, sondern sie auch zu praktischen Entschlüssen führt ..., doch wer ergreift diese Verantwortung? Wenn die Prospektive Pläne anbietet, die moralischen Erwartungen gerecht werden, wie etwa in diesem Fall, die Menschheit vor einer Großkatastrophe zu retten, stößt sie auf das Mysterium der menschlichen Freiheit angesichts von gut und böse. Die Prospektive scheint vorrangig nicht eine technische, sondern eine "menschliche" Aufgabe zu sein. Wenn wir 
eine wünschenswerte Zukunft vor Augen haben, so ist das wahre Problem nicht das ihrer Machbarkeit, sondern das ihrer ethischen Annehmbarkeit. Man muß eine Zukunft anstreben, die von allen verantwortet wird.

Auf dem Gebiet der Kommunikation zeigt uns die Prospektive ständig, wie die alten Modelle verfallen. Sie zerplatzen und sind in Krise. Dennoch läßt die Resignation der Mehrheit unter dem beherrschenden Joch der mächtigen Kommunikationsmittel erkennen, daß die Befreiung kommt, wenn neue Kommunikationsmodelle für die künftige Gesellschaft erstehen. Man muß damit anfangen, die Unzufriedenheit mit der technischen Unterdrückung, die uns manipuliert, spürbar zu machen, und Alternativen zum gegenwärtigen Kommunikationssystem entwerfen, aber nicht, indem man in der Gegenwart denkt, sondern indem man bereits im Herzen der Zukunft lebt. Man muß kreative Übungen vornehmen, ohne sich an die Grenzen der Zeit zu klammern.

\section{Die Kommunikation}

Wenn wir hier von Kommunikation sprechen, definieren wir sie als eine soziale Interaktion mittels Botschaften über die modernen Massenmedien. Es geht dabei um einen Prozeß wie um ein wirkliches Faktum, das eine neue Kultur hervorbringt. Und hier in Lateinamerika bildet dieser Kulturprozeß den Teil eines umfassenderen Systems, da wir, wenn wir über Kultur sprechen, das nicht unhistorisch tun können. Die Art und Weise, in der hier eine neue Welt entsteht, ist engstens verbunden mit den Kommunikationsmitteln, die innerhalb der Gesellschaft wirken. Und die Hersteller dieser Medien wissen sehr wohl um die Stoßkraft, deren Träger sie in der Welt und auf dem Sublontinent sind. Die elektronische Industrie erzeugt so, wie sie eine neue Wirtschaft mit sich bringt, auch eine neue Kultur. Die Hersteller der ferngesteuerten Bomben mit einer Fernsehkamera in deren Spitze, die es dem Piloten auf einem kleinen Fernsehschirm vorausschauend erlaubt, elektronisch von der Kabine aus die Ziele seiner zerstörerischen Sendung auszuwählen, sind dieselben, die auch die Fernsehgeräte in Serien herstellen. So führt uns das Sprechen über Kommunikation notwendig dazu, ihre konstitutiven Elemente innerhalb der gegenwärtigen Gesellschaftsstruktur aufzuspüren. Die Massenkommunikation ist eine Kulturindustrie, die Systeme von Schlüsseln und Regeln zur Steuerung von Menschengruppen schafft. Die Ideologie von Pato Donald oder von Popeye wie auch die Programme von Plan Cavisat (Audio-visuelles Zentrum über Satelliten) haben einen "normativen" Marktwert.

Die Erziehungstechnologie liegt in Händen großer internationaler Körperschaften, die auch die Erziehungsprogramme für Kinder und Arbeiter steuern. Die lateinamerikanischen Mitglieder von Instat (alle Länder Südamerikas), die ein System von 25 Satelliten besitzen, müssen hinnehmen, daß die USA (abgesehen davon, daß sie mehr als $90 \%$ der Gewinne einheimsen) die Technologie und die Programme beherrschen. Wie sagt doch A. Mattelart sehr gut: „In einer Welt, in der man nicht weiß, wer wer ist, wer die TV-Serie herstellt und wer die Rakete, fällt es schwer, die Kulturindustrie weiterhin als Leichtindustrie zu bezeichnen. ${ }^{\text {"3 }}$

Wenn wir auf die kommenden Jahre vorausschauen, scheinen die Kommunikationsmittel sich nicht nur immer stärker technologisch zu wandeln, sondern auch „kulturell", das heißt sie haben die Tendenz, ein immer stärker werdendes Ein-RichtungsWertsystem zu verteidigen und zu vereinheitlichen. Der Schauplatz der Welt wird 
aufgrund der von oben, von der Technologie her geweckten Wünsche gestaltet. Mit Sicherheit vermögen die Massenmedien die Art und Weise, menschliche Aufgabent in den Brennpunkt zu stellen, umzugestalten. Sie erzeugen Symbole und Massenbotschaften, sie schaffen durch Auswahl eine Bilderwelt innerhalb neuer Schemata. Sie gestalten ihre Publikumsgruppen über die Grenzen von Raum, Zeit und Sozialklassen hinweg. Mit einem Wort, die Massenmedien ermöglichen eine gesellschaftliche Interaktion großen Stils. In diesem Sinn haben sie das Wesen der Kultur qualitativ verändert. Jedoch stimmt, was ein Kommunikationsfachmann gesagt hat: "Alles ist in Bewegung, aber nichts wandelt sich", das heißt: Nichts wandelt sich außerhalb des Systems, außerhalb der modellhaft der Gesellschaft und dem Menschen aufgezwungenen Struktur. Die Zentren der Macht und die Wirtschaftsbasis beherrschen die Arterien der Massenkommunikation, indem sie immer stärker integrierte Gesellschaften schaffen. So entwickelt sich die Kommunikation voll und ganz zu einem politischen Problem, weil sie ein bestimmendes Element für die Haltungen des Gemeinlebens ist, für die Demokratiegestaltung und die politischen Konsensziele der Regierungen. Die Revolution der Kommunikation in der Erziehung, in der Industrie, in der Freizeit tendiert dahin, das Leben zu ver-technologisieren: Videokassetten in Farbe, Telefonuhren..., jedoch tendiert sie auch hin zur Kontrolle der sozialen Gesamtheit. Das Kommunikationsproblem ist mit der vorherrschenden Tätigkeit in der Gesellschaft insgesamt gekoppelt. Es ist der Zusammenhang des Gesamtsystems, der erschreckt. Die Konstrukteure des Fernsehens, der Röntgenstrahlen finanzieren auch das technologische Arsenal für die Folterung, für die Bürgerkontrolle, für die Förderung einer bestimmten „Kultur" und machen so andere ideologische Konstellationen unwirksam.

Die Politik der Kommunikation bewegt sich immer stärker auf eine politische Kommunikation $z$.

Aus diesem Grund verlaufen heute Kommunikation und Werbung sehr eng miteinander verbunden. Viele Wissenschaftler, Soziologen und Politiker machen sich Gedanken über die Aufgabe der Werbung, ein bestimmendes Element, wie man „Konsenshaltungen" erreicht. Schon im Jahre 1927 definierte Harold Lasswell die Werbung als die Handhabe der Kollektivhaltungen durch die Manipulation signifikativer Symbole.

In fast allen Ländern der Welt ergänzt die Werbung sehr häufig die physische Gewalt und die Einschüchterung. Der Bürger-Kunde wird nach dem Willen der "Macht" durch die Argumentation und die Oberzeugungskraft der Werbung eingefangen. Die Zukunft zeichnet sich ab als eine "totale Kommunikation der Macht", die noch verstärkt wird durch die schlichte Uberredung zum Konsum. Sie greift ein in die geistigen Strukturen, die Existenzmodelle, die Ideologien. Der Film „Fahrenheit $451^{\text {" }}$ ist bereits nicht mehr Science-fiction. Die Ordnungsmächte (Typ Chile, Uruguay, Brasilien ...) sind schon soweit und noch darüber hinaus.

Die Kontrolle der Kommunikation ist eins der großen Probleme, um die es sich ab sofort für die Zukunft zu mühen gilt, nicht nur theoretisch, sondern "politisch“. Der Prozeß der sozialen Kommunikation und die kulturelle und politische Dynamik bilden Teil eines und desselben Prozesses in dem verflochtenen Spiel der Mächte. Die Zukunft der Kommunikation in der Gesellschaft wird stark von ethischen Entscheidungen abhängen. Man muß die Produltion, die Organisation und die Strukturen der Botschaften innerhalb der Gesellschaft "menschlich" bestimmen. Der Wandel der Kulturgestaltung, der sozialen Beziehungen kommt aber nicht so sehr 
aus neuen Inhalten, sondern aus der technologischen Art, in der sie behandelt werden. Der kumulative Aufprall der von den gegenwärtigen (und den künftigen) Medien ausgestrahlten Botschaften besitzt die Kraft, jede soziale Ordnung zu erschüttern. Wenn wir also die soziale Kommunikation für die Zukunft bestimmen wollen, können wir die Tatsache nicht außer acht lassen, daß sie eine Struktur sozialer Beziehungen innerhalb eines bestimmten Ordnungsablaufs widerspiegelt. Wie werden sich heute... und morgen die technologischen Kommunikationsmittel und ihre Botschaften strukturieren?

\section{Lateinamerika, die Kommunikation und die Kirche}

Eine Prognose und Prospektive über die Aufgabe der Kirche in Lateinamerika hinsichtlich der Kommunikation ist erwiesenermaßen schwierig. Der riesige südamerikanische Subkontinent bietet sich dar als eine in höchstem Maß dem Wandel unterworfene Realität, die sehr schwer zu fassen ist. Die Politik, der wirtschaftliche und soziale Wandel gestalten die Kommunikation derart, daß man im Augenblick der Planung einer Aktion bereits mit weiteren Faktoren rechnen muß, die gerade erst auftauchen. Erst vor zehn Jahren, als Medellin fruchtbare Perspektiven für die pastorale Aktion eröffnete, schien die Kirche in eine neue historische Entscheidungsetappe einzutreten. Jetzt hingegen sehen wir, daß auch im Inneren der Kirche die Dinge sich völlig gewandelt haben. Wir sind $\mathrm{zu}$ vielen vorkonziliaren Auffassungen zurückgekehrt, und viele Bischöfe sehen mißtrauisch auf das, was sie selbst in Medellin unterzeichnet haben. Im politischen Bereich geschieht dasselbe. Uberall tritt der Militärstiefel gebieterisch die Erwartungen des Volkes nieder. Dadurch wird die Kommunikation in ihrem Wesen getroffen: die Menschen zueinander in Beziehung zu bringen. Von der Massenpresse bis hin zum Schulfernsehen übernimmt sie die Aufgabe, die finanziell-politisch-militärische Gesamtheit der Machthaber von heute zu rechtfertigen und zu verewigen. In Lateinamerika gibt es über 200 Fernsehkanäle, von denen mehr als 80 Prozent eisern durch die Militärzensur kontrolliert werden. Die große Presse befindet sich in den Händen mächtiger Privatunternehmen, die eng an den sie protegierenden Militärstaat gebunden sind.

In Ekuador wird die Filmwirtschaft von einer einzigen Person kontrolliert, die die Filme nach ihrem Gutdünken und offensichtlich unter Willkürzensur zuteilt. Der Rundfunk, das am meisten verbreitete Medium in Lateinamerika, könnte ein erzieherisches und evangelisierendes Instrument von guter Qualität abgeben. Zumindest erweist sich das an einigen Orten, an denen die lokalen Rundfunksender unter der Kontrolle kirchlicher Institutionen zur Förderung der Menschen arbeiten. Doch ist eine überwältigende Mehrheit von Rundfunksendern kommerziellen Charakters. Sie verbreiten Botschaften, um die Rundfunkhörer in das bereits etablierte Muster des Kulturkonsums zu ,integrieren“: Rundfunknachrichten, leichte Musik und Sport.

Die Kommunikation über Satelliten, die sich als immer stärkerer politischer Anreiz für Erziehungsprogramme abzeichnet, hängt in Lateinamerika von den großen nordamerikanischen Körperschaften $a b$, die in der Dritten Welt die Arten und Weisen „ausprobieren“, wie sie die armen Bevölkerungsgruppen in den Lebensstil der entwickelten Länder integrieren können. In dem Maße, wie die wissenschaftlichen Möglichkeiten den Kommunikationsstil im Übermaß verfeinern, erweist sich die Selbständigkeit und die Eigenkontrolle der Kommunikationsmittel schwieriger. Die großen ausländischen Unternehmensherren der Technologie stehen immer bereit, in Lateinamerika die neuesten Fortschritte einzusetzen. Der Blick in die Zukunft 
zeigt uns, daß die Kommunikationsmittel sich um einige wenige Polypen drängen, die Multinationalen, die in ihren Händen die Kontrolle vielfältiger Sektoren haben: von den Hotelketten angefangen über die Waffenfabriken und die Fernsehkanäle bis hin zur Karamellenherstellung. So ist die IT"T neben der telefonischen und telegrafischen Kommunikation in den verfeinerten Sektor eines ganzen Systems elektronischer Spürgeräte mittels Infrarotstrahlen eingedrungen, die in Kriegsfahrzeugen eingesetzt werden. Aber da der Vietnamkrieg ja zu Ende ist, ist jetzt dieselbe Technologie in den Händen unterdrückerischer Polizeikorps in den lateinamerikanischen Ländern.

In der gedruckten Kommunikation entwickelt sich das Zukunftspanorama auf derselben Linie. Die Papierherstellerfirmen werden immer stärker in sehr wenigen Händen kreolischer Magnaten oder aber ausländischer Firmen monopolisiert. Die Papiertrusts kontrollieren auch die Zeitschriften und Tageszeitungen. Die Fotoagenturen, die Magazine und die Zeitschriften, die den lateinamerikanischen Markt überschwemmen, sind Eigentum der Multinationalen (Rockefeller, Morgan, First National City Bank...) im Verbund mit ihren kreolischen Mitarbeitern. Augenfällig begünstigen die Millionen von Zeitschriften, die allmonatlich in alle Teile Lateinamerikas einströmen, nicht den Wandel, der erforderlich ist. Vielmehr impfen sie Konformismus ein, indem sie zeigen, daß ein Umsturz der bestehenden Verhältnisse nie gut ist, sondern daß die bessere Zukunft darin besteht, die Lebensmodelle der entwickelten Länder nachzuahmen.

Was wird also mit den Kommunikationsmitteln in den kommenden Jahren in Lateinamerika geschehen? Die Antwort, ich wiederhole es, ist nicht technologisch, sondern politisch. Es ist nutzlos, sich eine idealistische Sicht $\mathrm{zu}$ bilden und von einer

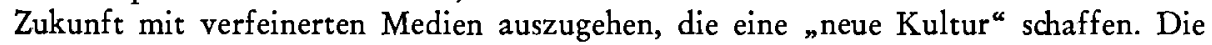
Kommunikationsmittel und die Rolle, die sie in der Gesellschaft spielen, wird abgegrenzt nach den historischen Umständen, nach der Fähigkeit der Menschen, eine Alternative $\mathrm{zu}$ unserem herrschaftsbestimmten System $\mathrm{zu}$ bilden. Alles andere ist Mechanikertum, ist Entfremdung.

Der bekannte Romanschreiber Fernando Medina Ferrada hat jüngst einen Roman geschrieben: "Los muertos están cada dia mas indóciles“ (Die Toten sind Tag für Tag unbelehrbarer). Er stellt das lateinamerikanische Volk als stetig unterdrückt dar, ohne anderen Ausweg, als „zwischen Erniedrigung und Tod“ zu wählen. Juan, die Hauptfigur, müde des Kampfes, das Volk gegen die Militäroligarchie zum Sieg zu führen, ist schon abgeschlagen. Die Wut, das blutige Schicksal, das er für die Massen erwartet, haben ihm den Träumerblick geraubt. Niemand lächelt, alle hoffen zu töten in einer Revolution, die nicht echt sein wird. Und trotzdem wird er ein neues Geschick für sein Land suchen, dïrstend nach Gerechtigkeit, immer geklammert an einen Strohhalm von Hoffnung...

Mir scheint, das kann ein Bild Lateinamerikas heute und in den kommenden Jahren abgeben. Ständig auf der Suche im Denken und im Handeln, was dem Volk selbst ermöglicht, die ganze Kommunikation in die Hände zu nehmen, auch die Technologie, um seine eigene Zukunft zu bauen.

Und was wird die Kirche tun? Welche Aufgabe hat sie? Die Kirche muß, ohne ihre Identität aufzugeben, immer stärker das leuchtende Gewissen des Volkes sein. Sie muß auch auf dem Gebiet der "Massenkultur" eine Kritik an dieser ermöglichen, um den wahren Problemkern der Kommunikation sichtbar zu machen, der mit den 
Infrastrukturen und dem ganzen ideologischen Apparat des Systems zusammenhängt, das diese unterstützt. Vom Evangelium her wird die Kirche ermöglichen, die Realität anders zu verstehen, auch die Kommunikation, ohne die Bereiche zu zerstückeln, sondern indem sie den neuerlichen Zusammenschluß zur Ganzheit aller Erscheinungsformen fördert, die letztlich die politische Wahrheit insgesamt bilden.

Aus dieser Sicht konkretisiert sich die Rolle der Kirche in der Kommunikation durch ein Handeln auf drei Ebenen:

- Auf der Ebene der Massenmedien in Händen kirchlicher Institutionen. Zur Zeit zeigt sich jedoch keine ermutigende Perspektive, da die Unterdrückung stark ist. Kein Rundfunksender oder Fernsehsender hat zu diesem Zeitpunkt (und möglicherweise auch in den kommenden Jahren) die Freiheit, die kulturelle Entfremdung anzuprangern, welche die wahre Kommunikation verhindert. Man muß sich innerhalb der Gleise behaupten, die durch Oligarchie und Militärmacht angezeigt sind.

- Auf der Ebene der Erziehungskommunikation. Die Kirche ist immer noch eine Kraft auf dem Gebiet der institutionalisierten Erziehung. In einigen Bereichen versucht man mit viel Erfolg, die Realität von einem neuen Kommunikationsstil her zu "lesen" (Plan Deni, Totale Sprache...). In der Zukunft kann die katholische Erziehung diese Tendenz fördern, sie stärker "geschichtsbildend“, tiefer eingebunden in die sozio-kulturelle Wirklichkeit, gestalten und der Kommunikation einen revolutionierenden Charakter verleihen. In außerschulischer, kommunitärer Erziehung kann sie Technologie in den Händen der Jugend einsetzen, damit diese „ihr Wort" spricht und lernt, sich des wissenschaftlichen Instruments der Kommunikation zu bedienen, und so eine andere Kultur schafft. Wenn sie es wollte, könnte die katholische Erziehung eine Speerspitze in der entsprechenden Seite des Systems sein.

- Auf der Ebene der Volkskommunikation. Die breite kirchliche Präsenz in verschiedenen Volksbereichen (Ortschaften, Gemeinschaften, Gruppen von Campesinos, Gruppen von Einheimischen ...) hat die Kommunikation mit Gruppenmedien entstehen lassen. Diese Art des Einsatzes und der Kommunikation erweist sich als ein Phänomen, das bei der Schaffung einer wirklichen Volkskultur zutiefst umgestaltend wirkt. Die Technologie wird noch auf viele Jahre in weiten Teilen des lateinamerikanischen Volks in den Anfängen bleiben. Wenn in der Stadt einige bereits das Kabelfernsehen benutzen, wird auf dem Land kaum das elektrische Licht aufleuchten. So gelten die (technologischen und nichttechnologischen) Gruppenmedien als Instrumente der Volkssprache, einer neuen Kommunikationspraxis. In der Zukunft wird das Anwachsen dieser Medien neue kulturelle Zusammenhänge zu schaffen ermöglichen.

Die Kirche ist noch immer eine Macht in Lateinamerika. Alles wird davon abhängen, wie sie diese Macht zur Befreiung des Volkes einsetzt. Auch zur Befreiung des Wortes und des Bildes, um den Mythos der Mächtigen anzukratzen, die die einzigen Kulturträger zu sein glauben.

Zum Teil hängt die Kommunikationszukunft des lateinamerikanischen Volkes von der Zulkunft der Kirche ab. Im Augenblick erschwert die Fortdauer des ideologischen Kampfes im Inneren der lateinamerikanischen Kirche die Einigung der Kräfte, um gegen die faschistischen Militärdiktaturen anzukämpfen. Wenn die Verantwortlichen der Kirche es fertig bringen, sich auf gemeinsame Ziele zu einigen, dann wird 
auch die Kommunikationspraxis sich ändern, sich auch befreit fühlen von gewissen Ketten, die ihre Botschaft einengen. Für die kommenden Jahre erweist sich eine Konfrontation zwischen der Kirche und der herrschenden Macht immer stärker als unvermeidbar. Die Harmonie wird nicht erreicht werden durch ideologische Nachgiebigkeit, sondern durch einen evangelischen, klaren und systematischen Kampf. Dadurch wird der Umsturz der bestehenden Verhältnisse eintreten. Der Wandel beginnt jedoch erst, wenn die Furcht ein Ende hat.

\section{Anmerkungen:}

1. Vgl. Agustin Merello: Prospectiva, Buenos Aires 1973, S. 96.

2. Lauro de Olivera Lima: La educación del futuro según McLuhan, Rio de Janeiro 1972, S. 14.

3. Aarmand Mattelart: La cultura como emprese multinacional, Mexico 1976, S. 17.

\section{S U M M A R Y}

Using the keywords ,prospective', ,communication' and ,Latin America ${ }^{c}$ the author gives an overview of the situation and the future of communications of the Church in Latin America. The imposition of restrictive measures from above might create more conflict. The future seems to be more a ,human' duty than one of technical development. Communications in Latin America tends more and more towards political communications in the hands of a few. The future of communications very much depends on ethical decisions. Unfortunately the structure of Communications media in Latin America is in the hands of multi-nationals and those few individuals who want to retain the existing system. The Church under these circumstances has only a few opportunities. She has only a few means of mass media at her disposal, especially in education and popular communications. Arguments within the Church at present hinder an evangelical, clear and systematic attempt to change the existing systems. This can only begin when Church leaders and responsible members are united and the internal struggle is ended.

\section{RÉS U MÉ}

L'auteur essaie, à l'aide des notions "prospective«, "communication « et "Amérique latine«, de réunir de façon concise les faits du sous-continent et de donner ici-même une vue d'ensemble résumant l'avenir de la communication dans l'Eglise. Il voit du danger dans le fait que des mesures coercitives, venant d'en haut, créent de nouveaux conflits. Pour lui, la prospective ne semble pas être en premier lieu, une tâche technique mais une tâche shumaine«. La politique de la communication, en Amérique latine, se meut de plus en plus fortement vers une communication politique, dominée par une oligarchie souveraine. L'avenir de la communication dépend fortement des décisions éthiques. Hélas! la structure des moyens de communication est dominée par les multinationaux et quelques indigènes qui ne font qu'accentuer les systèmes existants de l'oppression. Dans de telles conditions, l'Eglise n'a que des possibilités limitées. Avec l'aide de peu de mass media dans les mains de ses institutions, sur le plan de l'éducation, mais surtout sur le plan de la communication populaire. Des divergences d'opinions à l'intérieur de l'Eglise rendent, en ce moment, difficile un combat évangélique, clair et systématique pour une transformation des rapports existants, combat qui ne pourra commencer que lorsque les responsables de l'Eglise se seront mis d'accord et lorsque la crainte aura pris fin. 


\section{R E S U M E N}

Mediante los conceptos "prospectiva«, "comunicación " y "Latinoamérica el autor trata de presentar brevemente los hechos en el sub-continente y dar una visión panorámica del futuro de la comunicación de la Iglesia. Ve el peligro de que medidas impuestas desde arriba den lugar a nuevos conflictos. La prospección le parece prioritariamente como una misión "humana", no técnica. La política de la comunicación social en Latinoamérica se dirige cada vez más hacia una comunicación política, controlada por la oligarquía dominante. El futuro de la comunicación depende en gran medida de decisiones éticas. Desgraciadamente la estructura de la comunicación está dominada por las multinacionales y unos pocos autóctonos, que solo consolida el sistema de opresión existente. En estas circunstancias la Iglesia solo tiene posibilidades limitadas en el campo de la educación, sobre todo de la comunicación popular, gracias a unos pocos medios de difusión manejados por sus instituciones. Las controversias internas en la Iglesia dificultan actualmente una lucha evangélica, clara y sistemática para terminar con la situación existente, que solo podrá comenzar cuando los responsables de la Iglesia se pongan de acuerdo y se acabe con el miedo. 